VOL. 26 (1982), 45-56.

\title{
ON THE ESSENTIALLY-ALGEBRAIC THEORY GENERATED BY A SKETCH
}

\author{
G.M. KELLY
}

By a sketch we here mean a small category $S$ together with a small set $\Phi$ of projective cones in $S$, each cone $\phi \in \Phi$ being indexed by a small category $L_{\phi}$. A model of $S$ in any category $B$ is a functor $G: S \rightarrow B$ such that each $G \phi$ is a limit-cone. Let $F$ be any small set of small categories containing all the $L_{\phi}$. A small category $T$ admitting all F-limits (that is, an F-complete small $T$ ) is called an F-theory; it is considered as a sketch in which the distinguished cones are all the F-limit-cones. It is an important result of modern universal algebra, due originally to Ehresmann, that each sketch $S=(S, \Phi)$ with every $L_{\phi} \in F$ determines an $F$-theory $T$, with a generic model $M: S \rightarrow T$ of $S$, such that composition with $M$ induces an equivalence $M^{*}$ between the category of T-models in $B$ and that of $S$-models in $B$, whenever $B$ is $F$-complete. We give a simple proof of this result - one which generalizes directly to the case of enriched categories and indexed limits; and we make the new observation that the inverse to $M^{*}$ is given by (pointwise) right Kan extension along $M$.

\section{Introduction}

A category is said to be small if the set of isomorphism-classes of

Received 16 March 1982. The author gratefully acknowledges the assistance of the Australian Research Grants Committee. 
its objects is small. More generally, a set of objects in a category is loosely said to be small when it involves only a small set of isomorphismclasses. A projective cone $\phi: \Delta A \rightarrow P$ in a category $S$, where $P: L \rightarrow S$ and where $\Delta A: L \rightarrow S$ is the functor constant at $A$, is said to have indexing category $L$.

By a sketch we here mean a small category $S$ together with a small set $\Phi$ of projective cones in $S$, each $\phi \in \Phi$ having a small indexing category $L_{\phi}$. A functor $G: S \rightarrow B$ is called a model in $B$ of the sketch $(S, \Phi)$ if $G \phi$ is a limit-cone in $B$ for each $\phi \in \Phi$; these models determine a iull subcategory $\Phi-\operatorname{Mod}[S, B]$ of the functor-category $[S, B]$. It is well known (see [1], [4], and [8]) that purely algebraic objects of some kind in $B$, such as groups in $B$, are the models in $B$ of a suitable sketch; and that the same is true for certain non-purelyalgebraic objects, such as categories in $B$, which may be called "essentially algebraic".

Let $F$ be a small set of small categories. We call the sketch $(S, \Phi)$ an F-sketch if each indexing category $L_{\phi}$ is in $F$. An $F$-limit is one indexed by a category in $F$; a category $T$ is $F$-complete if it admits all F-limits, and then a functor $H: T \rightarrow B$ is F-continuous if it preserves these limits. A small F-complete category $T$ is called an F-theory. We identify such a theory with the sketch given by $T$ and all the F-limit-cones in $T$; so that a model of $T$ in $B$ is just an F-continuous $H: T \rightarrow B$. These models form the subcategory $\operatorname{Mod}[T, B]=F-\operatorname{Cts}[T, B]$ of $[T, B]$.

The following result on the existence of a "generic model" of a sketch is - except for the observation about Kan extensions - given in Bastiani and Ehresmann [1]:

THEOREM 1. Given an F-sketch $(S, \Phi)$, we can find an F-theory $T$ and a model $M: S \rightarrow T$ of $(S, \Phi)$ in $T$, with the following universal property: the functor $M^{*}: F-C t s[T, B] \rightarrow \Phi-\operatorname{Mod}[S, B]$, given by composition with $M$, is an equivalence of categories for every F-complete $B$. Moreover the equivalence-inverse of $M^{*}$ is given by (pointwise) right Kan extension along $M$.

The proof in [1] is a long one, carried out in several stages, each 
requiring an argument by transfinite induction; and does not yield much insight into the nature of $T$. Our aim in the present article is to give a simple proof using the notions of Kan extension and of density; and one moreover which extends directly to the case of enriched categories (with limits replaced by indexed limits). The enriched case will be dealt with in the final chapter of the author's forthcoming book [6]; what we give here is a streamlined proof in the classical case above, using only basic categorical ideas.

In the special case where $F$ is finite categories, a different proof is given in the thesis [2] of Coste, who starts from an axicnatic system in place of a sketch, and constructs $T$ syntactically in terms of formulas and provably-functional relations. It seems to the author that semantic arguments, such as are used in the present proof, are inherently simpler and more conceptual.

In the still more special case where $F$ is finite categories and $S$ is a Lawvere theory (with $\Phi$ being finite products), a very simple semantic proof is given by Kock and Reyes in [8]. In this case it is further observed by Kock in Appendix A of [7] that the inverse of $M$ is given by right Kan extension (but not that the extension is pointwise). The result that the inverse is so given in all cases seems to be new.

To avoid backing and filling between left and right Kan extensions, and between density and codensity, it turns out to be simpler to prove Theorem 1 in its dual form. The sketch $(S, \Phi)$ will be taken as $\left(A^{\circ p}, \Phi\right)$ below, with $A$ of course small. A functor $G: A \rightarrow B$ is a $\Phi$-comodel if each $G \phi$ is a colimit-cone in $B$; and these comodels form a full subcategory $\Phi-\operatorname{Com}[A, B]$ of $[A, B]$. The $F$-theory $T$ will appear as $D^{\circ p}$ for a certain $F$-cocomplete small $D$ to be constructed, and the generic model $M: S \rightarrow T$ will be $Z^{\text {op }}$, where $Z$ is a generic comodel $Z: A \rightarrow D$. Theorem 1 then asserts that, for an $F$-cocomplete $B$, the functor $Z^{*}: F-\operatorname{Cocts}[D, B] \rightarrow \Phi-\operatorname{Com}[A, B]$ is an equivalence with inverse Lan $_{Z}$, the (pointwise) left Kan extension along $Z$.

\section{Kan extensions and density}

We recall some elementary properties of these notions; many of them 
can be found in Mac Lane [9]. All the categories we consider are locally small: that is, their hom-sets are objects of the category Set of small sets. If $G: A \rightarrow B$ is any functor, we write $\tilde{G}: B \rightarrow\left[A^{\text {op }}\right.$, Set $]$ for the functor given by $\tilde{G} B=B\left(G_{-}, B\right)$.

Given functors $K: A \rightarrow C, S: C \rightarrow B$, and $G: A \rightarrow B$, there is, by two applications of the Yoneda lemma, a bijection between natural transformations $\alpha: G \rightarrow S K$ and transformations

$$
\alpha_{C B}^{\#}: B(S C, B) \rightarrow\left[A^{o p}, \operatorname{Set}\right](\tilde{K} C, \tilde{G} B)
$$

that are natural in both $C$ and $B$. We say that $S$ is the left Kan extension $\operatorname{Lan}_{K} G$ of $G$ along $K$, with unit $\alpha$, if $\alpha^{\#}$ is an

isomorphism. The extension exists precisely when $\left[A^{\circ p}\right.$, Set $]\left(\tilde{K} C, \tilde{G}^{-}\right)$is representable as $B(S C,-)$ for each $C$; and the extension is then unique to within isomorphism, since this representation is so. The extension certainly exists whenever $A$ is small and $B$ is cocomplete, for then we get the desired representation by setting $S C$ equal to the coend $\int^{A} \mathrm{C}(K A, C) \cdot G A$, where the integrand denotes the coproduct of $\mathrm{C}(K A, C)$ copies of $G A$. The term "extension" is somewhat misleading, since the unit $\alpha$ need not be an isomorphism; it is easy however to see that $\alpha$ is an isomorphism if $K$ is fully faithful.

When $\operatorname{Lan}_{K} G$ exists, we have for any functor $R: C \rightarrow B$ an isomorphism

$$
[C, B]\left(\operatorname{Lan} K^{G}, R\right) \cong[A, B](G, R K)
$$

sending $\beta: \operatorname{Lan}_{K} G \rightarrow R$ to $\beta K \cdot \alpha ;$ for to give the components ${ }^{B} C:\left(\operatorname{Lan}_{K} G\right) C \rightarrow R C$ is, by our definition of the Kan extension, to give maps $\bar{\beta}_{C}: \tilde{K} C \rightarrow \tilde{G} R C$ natural in $C$, or equivalently maps $\bar{\beta}_{A C}: C(K A, C) \rightarrow B(G A, R C)$ natural in $A$ and $C$; which by Yoneda is to give $\gamma_{A}: G A \rightarrow R K A$, or $Y: G \rightarrow R K$.

The universal property ( 1 ) is taken by many authors as the definition of left Kan extension, but is in fact strictly weaker than our definition. 
Mac Lane [9] calls our stronger notion the pointwise left Kan extension; we prefer our present terminology because "non-pointwise" Kan extensions seem to be totally useless in mathematics.

If $\operatorname{Lan}_{K} G$ exists with unit $\alpha$, and if $Q: B \rightarrow D$ has a right adjoint $P$, then $Q \operatorname{Lan}_{K} G$, with unit $Q \alpha$, is $\operatorname{Lan}_{K}(Q G)$; this follows easily from our definition, when we observe that $(Q G)^{\sim} D \cong \tilde{G} P D$.

We call a functor $K: A \rightarrow C$ dense if $\tilde{K}: C \rightarrow\left[A^{o p}\right.$, Set $]$ is fully faithful. Since this is the assertion that $\tilde{K}$ gives an isomorphism $\mathcal{C}(C, D) \rightarrow\left[A^{\mathrm{op}}, \operatorname{Set}\right](\tilde{K} C, \tilde{K} D)$, it is equivalent to the assertion that $\operatorname{Lan}_{K} K=I_{C}$, with the identity as unit.

Such a dense $K$ gives an equivalence between $C$ and the full subcategory $C^{\prime}$ of $\left[A^{O P}\right.$, Set $]$ formed by the objects isomorphic to some $\tilde{K} C$. The Yoneda isomorphism

$$
\left[A^{\text {op }} \text {, Set }\right](A(-, A), \tilde{K} C) \cong(\tilde{K} C) A=C(K A, C) \cong C^{\prime}(\tilde{K} K A, \tilde{K} C)
$$

exhibits the object $\tilde{K} K A$ of $C^{\prime}$ ' as the reflexion into $C^{\prime}$ ' of the representable $A(-, A)$ of $\left[A^{\circ p}\right.$, Set $]$; and the unit of this reflexion is the map $K_{-A}: \mathrm{A}(-, A) \rightarrow \mathcal{C}(K-, K A)=\tilde{K} K A$. Hence $\mathcal{C}^{\prime}$ contains the representables if and only if this unit is an isomorphism for each $A$ : which is exactly to say that $K$ is fully faithful.

PROPOSITION 2 (cf. Diers [3]). Let the dense $K: A \rightarrow C$ be the composite of $Z: A \rightarrow D$ and $J: D \rightarrow C$, where $J$ is fully faithful. Then we have $\tilde{Z} \cong \tilde{K} J$; both $Z$ and $J$ are dense; if some Lan ${ }_{K} G$ exists with unit $\alpha$, then $\operatorname{Lan}_{Z} G=\left(\operatorname{Lan}_{K} G\right) J$, again with wit $\alpha$; and in particular $\operatorname{Lan}_{Z} K=J$ with the identity as unit.

Proof. We have

$$
\tilde{K}_{J} D=\mathcal{C}(K-, J D)=C\left(J Z_{-}, J D\right) \cong C(Z-, D)=\tilde{Z} D,
$$

giving $\tilde{K} J:: \tilde{Z}$. Since $\tilde{K}$ and $J$ are fully faithful, so is $\tilde{Z}$; hence $Z$ is dense. For any $G: A \rightarrow B$ such that $\operatorname{Lan}_{K} G$ exists, we have 


$$
\left[A^{\mathrm{op}}, \operatorname{Set}\right](\tilde{Z} D, \tilde{G} B)=\left[A^{\mathrm{op}}, \operatorname{Set}\right](\tilde{K} J D, \tilde{G} B) \cong B\left(\left(\operatorname{Lan}_{K} G\right) J D, B\right),
$$

so that $\operatorname{Lan}_{Z} G=\left(\operatorname{Lan}_{K} G\right) J$. Since $\operatorname{Lan}_{K} K={ }^{I_{C}}$ this gives $\operatorname{Lan}_{Z} K=J$. From this we get

$$
\tilde{J} C=\mathcal{C}\left(\left(\operatorname{Lan}_{Z^{K}}\right)-, C\right) \cong\left[A^{\mathrm{op}}, \operatorname{Set}\right]\left(\tilde{z}_{-}, \tilde{K} C\right)
$$

To give a map $\beta: \tilde{J} C^{\prime} \rightarrow \tilde{J} C$ with components

$$
\beta_{D}: C\left(J D, C^{\prime}\right) \rightarrow(\tilde{J} C) D \cong\left[A^{o p}, \operatorname{Set}\right](\tilde{Z} D, \tilde{K} C)
$$

is therefore to give components

$$
\beta_{D A}: C\left(J D, C^{\prime}\right) \rightarrow \operatorname{Set}(D(Z A, D), C(K A, C))
$$

natural in both $D$ and $A$; or equivalently components

$$
\bar{\beta}_{D A}: D(Z A, D) \rightarrow \operatorname{Set}\left(C\left(J D, C^{\prime}\right), \mathcal{C}(K A, C)\right)
$$

natural in $D$ and $A$, which by Yoneda is to give maps $\gamma_{A}: \mathcal{C}\left(J Z A, C^{\prime}\right) \rightarrow \mathcal{C}(K A, C)$ natural in $A$. Since $K=J Z$ is dense, such a $\gamma$ is $\mathcal{C}(K A, f)$ for a unique $f: C^{\prime} \rightarrow C$. It follows that $\beta$ is $\tilde{J} f$ for a unique $f$, so that $\tilde{J}$ is furly faithful and $J$ is dense.

\section{The category of $\Phi$-algebras}

From now on $\left(A^{\circ p}, \Phi\right)$ is a given F-sketch. By an $\left(A^{\circ p}, \Phi\right)$-algebra, or a $\Phi$-algebra for short, we mean a model of $\left(A^{\circ p}, \Phi\right)$ in Set. We write $C$ for the category $\Phi-A l g=\Phi-\operatorname{Mod}\left[A^{o p}\right.$, Set $] \subset\left[A^{o p}\right.$, Set $]$, and $I: C \rightarrow\left[A^{\circ p}\right.$, Set $]$ for the full inclusion. Using a simple argument by transfinite induction, Gabriel and Ulmer ([4], Satz 8.5) show that $I$ has a left adjoint $R$. This result is the kernel of our proof of Theorem 1 ; for all we use apart from it are elementary categorical considerations. (The same is true in the enriched case [6]; but there the existence of the left adjoint $R$ uses the more subtle transfinite-induction arguments of [5].) It follows that the category $\mathrm{C}$ of $\Phi$-algebras is complete and cocomplete.

We write $K: A \rightarrow C$ for the composite of the reflexion 
$R:\left[A^{\text {op }}\right.$, Set $] \rightarrow C$ and the Yoneda embedding $Y: A \rightarrow\left[A^{\text {op }}\right.$, Set $]$. Then we have

$$
\tilde{K} C=\mathcal{C}\left(K_{-}, C\right)=\mathcal{C}\left(R Y_{-}, C\right) \cong\left[A^{o p}, \operatorname{Set}\right]\left(Y_{-}, I C\right) \cong I C ;
$$

so that $\tilde{K}$ is (to within isomorphism) the full inclusion $I$, and $K$ is therefore dense.

The condition for $G: A \rightarrow B$ to be a comodel can be expressed directly in terms of $K$. For $G \phi$ is a colimit-cone in $B$ exactly when $b^{\prime}(G \phi, B)=(\tilde{G} B) \phi$ is a limit-cone in Set for each $B \in B$; that is, when $\tilde{G} B$ is a $\Phi$-algebra for each $B$. Thus:

PROPOSITION 3. $G: A \rightarrow B$ is an $\left(A^{\text {Op }}, \Phi\right)$-comodel exactly when $\tilde{G}: B \rightarrow\left[A^{o p}\right.$, Set $]$ factorizes through the fully-faithful $\tilde{K}: \mathcal{C} \rightarrow\left[A^{\mathrm{op}}\right.$, Set] as $\tilde{G} \cong \tilde{K} T$. In particular $K: A \rightarrow C$ is itself a comodel.

As we saw in Section $2, K$ is fully faithful precisely when the representables $A(-, A)$ are $\Phi$-algebras; which in turn is exactly to say that each cone $\phi$ is a colimit-cone in $A$, or a limit-cone in $A^{\circ p}$. In this case, of course, $K$ is just the Yoneda embedding $Y$ seen as landing in $\mathcal{C}$, and we have $\tilde{K} K \stackrel{=}{=}$. This applies in particular when $A$ is a small F-cocomplete category, and we take for $\Phi$ all the $F$-colimits in $A$, so that $\left(A^{O P}, \Phi\right)$ is the F-theory $A^{O P}$. Since the category of algebras is now F-Cts $\left[A^{\circ P}\right.$, Set] , since the comodel $K$ preserves F-colimits, and since $K$ is fully faithful, we have:

PROPOSITION 4. For a small F-cocomplete $A$, the full inclusion $K: A \rightarrow F-C t s\left[A^{\circ p}\right.$, Set $]$ preserves and reflects F-colimits.

We now return to a general sketch $\left(A^{\circ p}, \Phi\right)$ as before, with $C=\Phi-A l g$ and $K: A \rightarrow C$ the canonical comodel in $C$. For any $B$, we write $\operatorname{Ladj}[C, B]$ for the full subcategory of $[C, B]$ given by those $S: C \rightarrow B$ which are left adjoints. Since such an $S$ preserves all colimits and since $K$ is a $\Phi$-comodel, so is $S K$. Hence composition with $K$ induces a functor $K^{*}: \operatorname{Lad} j[C, B] \rightarrow \Phi-\operatorname{Com}[A, B]$. 
PROPOSITION 5. For any $S \in \operatorname{Ladj}[C, B]$ we have $\operatorname{Lan}_{K}(S K)=S$, with unit the identity. $A$-comodel $G: A \rightarrow B$ is isomorphic to some $S K$ with $S \in \operatorname{Ladj}[C, B]$ if and only if $\operatorname{Lan}_{K} G$ exists. Thus $K^{*}$ gives an equivalence between $\operatorname{Lad} j[C, B]$ and the fulz subcategory $\Phi-\operatorname{Com}[A, B]$ ' given by such comodels; and the inverse of this equivalence is given by left Kan extension along $K$.

Proof. Since $K$ is dense, we have $\operatorname{Lan}_{K} K=1_{C}$ with the unit as identity; therefore, as we saw in Section 2, for a left adjoint $S$ we have $\operatorname{Lan}_{K}(S K)=S$ with unit the identity. Now let $G$ be any $\Phi$-comodel for which $\operatorname{Lan}_{K} G$ exists. We are to show that $S=\operatorname{Lan}_{K} G$ is left adjoint and that $S K \cong G$. Since $G$ is a $\Phi$-comodel we have $\tilde{G} \cong \tilde{K} T$ for some $T$ by Proposition 3. By the definition of Kan extension we have

$$
B(S C, B) \cong\left[A^{\circ \mathrm{p}}, \operatorname{Set}\right](\tilde{K} C, \tilde{G} B) \cong\left[A^{\circ \mathrm{p}}, \operatorname{Set}\right](\tilde{K} C, \tilde{K} T B) ;
$$

and this is isomorphic to $\mathcal{C}(C, T B)$ since $\tilde{K}$ is fully faithful. Hence $S \rightarrow T$. Moreover we have

$$
B(S K A, B) \cong C(K A, T B)=(\tilde{K} T B) A \cong(\tilde{G} B) A=B(G A, B),
$$

whence we have $S K \cong G$.

We in fact use this below only when $B$ is cocomplete. In that case $\operatorname{Lan}_{K} G$ exists for every $G$, and the left-adjoint functors $S: \mathcal{C} \rightarrow B$ are exactly the cocontinuous ones (since $C$ has a small dense subcategory see, for example, [6], Theorem 5.33). So $K^{*}$ and $\operatorname{Lan}_{K}$ provide an equivalence between $\operatorname{Cocts}[C, B]$ and $\Phi-\operatorname{Com}[A, B]$; which is (in dual form) the analogue of Theorem 1 when $F$ is replaced by "all small categories".

4. The theory $D^{\text {OP }}$ and the equivalence $D^{\text {OP }}-A 1 g \simeq \Phi-A 1 g$

We define $D$ to be the closure under F-colimits, in the cocomplete category $C=\Phi-A l g$, of the full subcategory $D_{0}$ given by the objects $K A$ with $A \in A$. We can construct $D$ by transfinite induction: with $D_{0}$ as given, we set $D_{\alpha+1}$ equal to the full subcategory given by all $F$-colimits 
of diagrams in $D_{\alpha}$, and set $D_{\alpha}=\underset{\beta<\alpha}{U} D_{\beta}$ at a limit-ordinal $\alpha$. Since $F$ is small and $A$ is small, each $D_{\alpha}$ is small. The sequence $\left(D_{\alpha}\right)$ is clearly stationary from $D_{\gamma}$ on, where $\gamma$ is a regular cardinal exceeding the number of objects in each $L \in F$. Thus $D=D_{\gamma}$ is itself small. Clearly it is the smallest replete full subcategory of $\mathcal{C}$ containing all the $K A$ and closed under F-colimits; moreover it does admit all F-colimits since $C$ is cocomplete, and the full inclusion $J: D \rightarrow C$ preserves and reflects these. We write $K=J Z$ for the factorization of $K: A \rightarrow C$ through $J$. Since $K$ is a $\Phi$-comodel by Proposition 3, so is $Z$ by the remarks above about $J$. By Proposition 2 , both $Z$ and $J$ are dense.

PROPOSITION 6. The replete image of the fully-faithful

$\tilde{J}: \mathcal{C} \rightarrow\left[D^{\circ \mathrm{p}}\right.$, Set $]$ is the full subcategory F-Cts $\left[0^{\circ \mathrm{p}}\right.$, Set $]$ given by the algebras for the F-theory $D^{\mathrm{op}}$.

Proof. Since $J$ preserves F-colimits, each $\tilde{J} C=C\left(J_{-}, C\right)$ sends them to limits in Set, as required. For the converse, let $P: D^{\circ p} \rightarrow$ Set be $F$-continuous; we are to show that $P$ is isomorphic to some $\tilde{J} C$. Since $Z: A \rightarrow D$ is a $\Phi$-comodel and $P^{\mathrm{op}}: D \rightarrow$ Set $^{\mathrm{op}}$ is F-cocontinuous, $P^{\mathrm{OP}} Z: A \rightarrow$ Set $^{\mathrm{OP}}$ is a $\Phi$-comodel. Since Set $^{\mathrm{OP}}$. is cocomplete, $\operatorname{Lan}_{K}\left(P^{\left.\circ p_{Z}\right)}\right.$ exists; and if this is $S: \mathcal{C} \rightarrow \operatorname{Set}^{\circ p}$, then, by Proposition 5, $S$ is left adjoint, and the unit $\alpha: P^{\mathrm{op}_{Z}} \rightarrow S K$ of $\operatorname{Lan}_{K}\left(P^{\circ p_{Z}}\right)$ is an isomorphism. By Proposition 2 we have $\operatorname{Lan}_{Z}\left(P^{\circ p_{Z}}\right)=S J$ with unit the isomorphism $\alpha$. By (1) the identity $1: P^{\mathrm{op}_{Z}} \rightarrow P^{\mathrm{op}_{Z}}$ is $\beta Z . \alpha$ for some $\beta: S J \rightarrow P^{\text {op }}$. We claim that $\beta$ is an isomorphism. To see this, let $D^{\prime}$ be $\left\{D \in D \mid B_{D}\right.$ is an isomorphism $\}$. Then $D^{\prime}$ contains each $Z A$ since $B Z$ is the isomorphism $\alpha^{-1} ;$ and $D^{\prime}$ is closed in $D$ under F-colimits, since both $S J$ and $P^{\text {op }}$ are F-cocontinuous: so that $D^{\prime}$ is all of $D$. Thus $P^{\mathrm{Op}} \cong S J$. Since a left adjoint $S: \mathcal{C} \rightarrow$ Set $^{\text {op }}$ is necessarily given by $S^{\mathrm{OP}} \cong \mathrm{C}(-, C)$ for some $C \in \mathcal{C}$, we have 
$P \cong C(J-, C)=\tilde{J} C$, as required.

This last proposition is in effect the case $B=$ Set of Theorem 1 . We now pass to the general case.

\section{Proof of the theorem}

For any $F$-cocomplete $B$ and any $F$-cocontinuous $H: D \rightarrow B$, the composite $H Z: A \rightarrow B$ is a $\Phi$-comodel since $Z$ is; so that composition with $Z$ gives a functor $Z^{*}: F-\operatorname{Cocts}[D, B] \rightarrow \Phi \rightarrow \operatorname{Com}[A, B]$. We are to show $Z^{*}$ to be an equivalence, with inverse the restriction of $\operatorname{Lan}_{Z}$.

We first show that, for any $F_{-c o c o n t i n u o u s ~} H$, we have $\operatorname{Lan}_{Z}(H Z) \cong H$. Since $H$ is a comodel for the theory $D^{\circ p}$, and since the full inclusion $D^{\circ p}-A l g \rightarrow\left[D^{o p}\right.$, Set $]$ is "equivalent" to $\tilde{J}$ by Proposition 6 , it follows from Proposition 3 that $\tilde{H} \cong \tilde{J} T$ for some $T: B \rightarrow C$. If we set $G=H Z$, we have

$$
\tilde{G} B=B\left(H Z_{-}, B\right)=(\tilde{H} B) Z \cong(\tilde{J} T B) Z=\mathcal{C}\left(J Z_{-}, T B\right)=\mathcal{C}\left(K_{-}, T B\right)=\tilde{K} T B .
$$

Using $\tilde{Z} \cong \tilde{K} J$ from Proposition 2, we have since $\tilde{K}$ is fully faithful that

$$
\begin{aligned}
& {\left[A^{\circ p}, \operatorname{Set}\right](\tilde{Z} D, \tilde{G} B) \cong\left[A^{\circ p}, \operatorname{Set}\right](\tilde{K} J D, \tilde{K} T B) \cong \mathcal{C}(J D, T B) } \\
&=(\tilde{J} T B) D \cong(\tilde{H} B) D=\mathcal{C}(H D, B) ;
\end{aligned}
$$

which gives the desired result $\operatorname{Lan}_{Z} G \cong H$.

The proof will now be complete when we show that every $\Phi$-comodel $G: A \rightarrow B$ is isomorphic to $H Z$ for some F-cocontinuous $H: D \rightarrow B$. We first do this in the case where $B$ is small, so that we can look on $B^{\text {op }}$ itself as an F-theory. Then by Proposition 4 we have a fully-faithful $L: B \rightarrow F-C t s\left[B^{\circ p}\right.$, Set] that preserves and reflects F-colimits. Hence $L G$ is a $\Phi$-comodel since $G$ is. Moreover $\operatorname{Lan}_{K}(L G)$ exists, since the category $E=F-C t s\left[B^{\circ p}\right.$, Set $]$ of $B^{\circ p}$-algebras is cocomplete. By Proposition 5, therefore, we have $L G \cong S K$ for some left-adjoint $S: C \rightarrow E$. Set

$$
D^{\prime}=\{C \in \mathcal{C} \mid S C \cong L B \text { for some } B \in B\} .
$$

Then $D^{\prime}$ contains each $K A$ since $S K A \cong L G A$, and $D^{\prime}$ is closed under 
F-colimits since $S$ and $L$ preserve these. Therefore $D^{\prime}$ contains $D$, so that $S J \cong L H$ for some $H: D \rightarrow B$; and $H$ is $F$-cocontinuous since $S J$ is and since $L$ reflects F-colimits. Moreover, since $L H Z \cong S J Z=S K \cong L G$ and since $L$ is fully faithful, we have $H Z \cong G$, as required.

If $B$ is large, we define the full subcategory $B^{\prime}$ of $B$ as the closure in $B$ under F-colimits of the objects of the form $G A$. Then $B^{\prime}$ is small since $A$ and $F$ are small, and the full inclusion $N: B^{\prime} \rightarrow B$ preserves and reflects F-colimits. If the factorization of $G$ through $N$ is $N G^{\prime}$, then $G^{\prime}$ is again a $\Phi$-comodel, so that by the argument above $G^{\prime} \cong H^{\prime} Z$ for some $F$-cocontinuous $H^{\prime}$. Now we have $G \cong H Z$ where $H$ is the F-cocontinuous $N H^{\prime}$. This completes the proof.

\section{References}

[1] Andrée Bastiani and Charles Ehresmann, "Categories of sketched structures", Cahiers Topologie Géom. Différentielze 13 (1972), $103-214$.

[2] M. Coste, "Localisation dans les catégories de modèles" (Thesis, Université Paris Nord, Paris, 1977).

[3] Yves Diers, "Type de densité d'une sous-catégorie pleine", Ann. Soc. Sci. Bruxelzes Sér. I 90 (1976), 25-47.

[4] Peter Gabriel, Friedrich Ulmer, Lokal präsentierbare Kategorien (Lecture Notes in Mathematics, 221. Springer-Verlag, Berlin, Heidelberg, New York, 1971).

[5] G.M. Kelly, "A unified treatment of transfinite constructions for free algebras, free monoids, colimits, associated sheaves, and so on", Bulz. Austral. Math. Soc. 22 (1980), 1-83.

[6] G.M. Kelly, Basic concepts of enriched category theory (London Mathematical Society Lecture Notes, 64. Cambridge University Press, London, New York, Sydney, 1982).

[7] Anders Kock, Synthetic differential geometry (London Mathematical Society Lecture Notes, 51. Cambridge University Press, London, $1981)$. 
[8] A. Kock, G.E. Reyes, "Doctrines in categorical logic", Handbook of mathematical Logic, 283-313 (studies in Logic and the Foundations of Mathematics, 90. North-Holland, Amsterdam, New York, Oxford, 1977 ).

[9] S. Mac Lane, Categories for the working mathematician (Graduate Texts in Mathematics, 5. Springer-Verlag, New York, Heidelberg, Berlin, 1971).

Department of Pure Mathematics, University of Sydney,

Sydney,

New South Wales 2006,

Australia. 